\title{
Balloon pulmonary angioplasty after pulmonary thromboendarterectomy
}

\author{
Massimiliano Palazzini, Francesco Saia, Nevio Taglieri, Daniele Guarino, Mariangela Rotunno, \\ Nazzareno Galiè, Fabio Dardi \\ Alma Mater Studiorum, University of Bologna and IRCCS Sant'Orsola University Hospital, Bologna, Italy \\ Correspondence to: Massimiliano Palazzini. Alma Mater Studiorum, University of Bologna and IRCCS Sant'Orsola University Hospital, Bologna, Italy. \\ Email: massimiliano.palazzini@unibo.it.
}

Submitted Oct 25, 2021. Accepted for publication Jan 10, 2022.

doi: 10.21037/acs-2021-pte-19

View this article at: https://dx.doi.org/10.21037/acs-2021-pte-19

\section{Background}

Pulmonary thromboendarterectomy (PTE) is the treatment of choice for patients with chronic thromboembolic pulmonary hypertension (CTEPH) as it can remove the chronic, fibrotic, flow-limiting organized thrombi within the pulmonary arterial bed, addressing the primum movens of the disease (1). Despite significant improvement in all haemodynamic parameters, residual pulmonary hypertension $(\mathrm{PH})$ is frequent after PTE, ranging from $17 \%$ to $31 \%(2,3)$. There is no clear definition of residual $\mathrm{PH}$ after PTE, and the actual incidence of this condition has been difficult to quantify. Usually, moderate residual $\mathrm{PH}$ is well tolerated by patients and, as shown by data from the United Kingdom cohort, clinically relevant residual PH after PTE mainly occur when the mean pulmonary arterial pressure (mPAP) is greater than $30-35 \mathrm{mmHg}$ (3). The risk of persistent/recurrent $\mathrm{PH}$ in the long-term underlines the importance of a systematic patient followup, even after PTE. Balloon pulmonary angioplasty (BPA) has been developed as a compassionate procedure for symptomatic patients with CTEPH who are ineligible for surgery or with persistent/recurrent $\mathrm{PH}$ after PTE. BPA is not able to remove clots as PTE, but it is able to restore the flow by fragmenting the thrombotic and fibrotic material, resulting in hemodynamic and clinical improvement. Selection of good candidates for BPA, especially after PTE, includes a complete re-assessment of the patient with persistent symptomatic PH after PTE at least four to six months after surgery using high quality imaging techniques such as computed tomography pulmonary angiography (CTPA), selective pulmonary angiography (to provide fine details) and right heart catheterization (RHC) to assess the hemodynamic impairment. However, these imaging techniques are not widely available and require expertise.

\section{Clinical vignette}

A sixty-one-year-old male was referred to our centre in September 2020 for exertional dyspnoea and severe right chambers enlargement on the echocardiogram. The patient had been treated with warfarin with sub therapeutic values of international normalized ratio (INR) (self-managed) since the diagnosis of acute pulmonary embolism in 2005. CTPA and non-selective pulmonary angiography showed bilateral perfusion defects suggestive of chronic thromboembolism while RHC confirmed severe pre-capillary $\mathrm{PH}$ [right atrial pressure (RAP) $7 \mathrm{mmHg}$; mPAP $60 \mathrm{mmHg}$; pulmonary artery wedge pressure (PAWP) $8 \mathrm{mmHg}$; cardiac index (CI) $3.0 \mathrm{~L} / \mathrm{min} / \mathrm{m}^{2}$; pulmonary vascular resistance (PVR) 8.4 woods units (WU)].

The case was discussed within a multidisciplinary team (MDT), and the patient was deemed suitable for PTE, which was done on November 2020. Due to haemodynamic impairment sildenafil therapy, $20 \mathrm{mg}$ three times a day (TID) was started as bridging for surgery and withdrawn after PTE. Mild improvement of symptoms after surgery was documented during follow-up outpatient visits.

In February 2021, CTPA and RHC were repeated, as per our centres standard clinical practice for all patients three to six months after PTE. RHC showed the persistence of $\mathrm{PH}$ (RAP $8 \mathrm{mmHg}$; mPAP $51 \mathrm{mmHg}$; PAWP $10 \mathrm{mmHg}$; CI $2.5 \mathrm{~L} / \mathrm{min} / \mathrm{m}^{2}$; PVR $8.0 \mathrm{WU}$ ) and CTPA showed continual 
bilateral, multiple sub-segmental perfusion defects (endoluminal webs). Sildenafil therapy was recommenced at $20 \mathrm{mg}$ TID, and the anatomic lesions were deemed suitable for BPA following further MDT discussion.

The patient underwent three BPA sessions during which segments seven and ten (May 2021), segments four and eight (June 2021) of the right lung and segment 10 (November 2021) of the left lung were treated. After these sessions, the patient improved significantly in terms of symptom reduction and hemodynamics, which showed a reduction of PVR from 8.0 to 5.4 WU. Further sessions are planned.

\section{Technique}

Percutaneous vascular access for BPA was performed via the femoral vein, using long sheaths through which right coronary artery catheters were inserted. Oral anticoagulant therapy with vitamin $\mathrm{K}$ antagonist was withdrawn at least five days before the procedure and replaced with low molecular weight heparin (when the INR was $<2.0$ ). A biplane angiography catheter laboratory was used to provide simultaneous anterior-posterior and lateral projections, and to avoid excessive exposure to iodized contrast medium. Three-dimensional reconstructions of the pulmonary tree by CTPA were also used to help the operators, two interventional cardiologists, recognize and selectively cannulate the vessels to be treated. In BPA, use of undersized balloons is initially recommended to reduce reperfusion injury and to allow vessels to gradually regain the original size before optimizing results with larger balloons. During the procedure unfractionated heparin was administered, monitoring the activated clotting time (target value $200-250$ s).

\section{Comments and conclusions}

Evidence in the efficacy of BPA after PTE is lacking. Shimura et al. described BPA in nine patients who had undergone PTE but had gradually deteriorated after an initially favourable response (4). Hemodynamics and functional class improved at follow-up and the authors recommend combined PTE and BPA for patients with both proximal and very distal lesions (4). In our experience, between 2015 and 2020, nine patients (mean age $59 \pm 11$ years; five females) with persistent $\mathrm{PH}$ after PTE underwent BPA. After an average of two sessions, we observed a hemodynamic improvement (reduction of mPAP from $44 \pm 10$ to $38 \pm 10 \mathrm{mmHg}$; reduction of PVR from $6.6 \pm 2.2$ to $6.3 \pm 4.6 \mathrm{WU}, \mathrm{P}$ value $>0.05$ ). The hemodynamic improvement in this small subset of patients appears less evident in comparison with surgical naïve patients [43 patients (mean age $69 \pm 14$ years; female 24 ); reduction of mPAP from $43 \pm 14$ to $35 \pm 11 \mathrm{mmHg}$ (P value $<0.05$ ) and reduction of PVR from $7.5 \pm 3.5$ to $5.2 \pm 3.2 \mathrm{WU}, \mathrm{P}$ value $<0.05$, after a median of 3 sessions]. A possible explanation could be that surgically treated vessels have an abnormal compliance and their recoil could be a limiting factor, ending in poor angiographic and haemodynamic results.

We can conclude that the role of BPA after PTE is to treat distal web-like lesions that cannot be reached by the surgeon. Albeit this procedure seems to be able to improve symptoms and hemodynamics, the selection of eligible patients, although mandatory, is not easy, especially after PTE.

\section{Acknowledgments}

Funding: None.

\section{Footnote}

Conflicts of Interest: The authors have no conflicts of interest to declare.

Open Access Statement: This is an Open Access article distributed in accordance with the Creative Commons Attribution-NonCommercial-NoDerivs 4.0 International License (CC BY-NC-ND 4.0), which permits the noncommercial replication and distribution of the article with the strict proviso that no changes or edits are made and the original work is properly cited (including links to both the formal publication through the relevant DOI and the license). See: https://creativecommons.org/licenses/by-nc-nd/4.0/.

\section{References}

1. Galiè N, Humbert M, Vachiery JL, et al. 2015 ESC/ERS Guidelines for the diagnosis and treatment of pulmonary hypertension: The Joint Task Force for the Diagnosis and Treatment of Pulmonary Hypertension of the European Society of Cardiology (ESC) and the European Respiratory Society (ERS): Endorsed by: Association for European Paediatric and Congenital Cardiology (AEPC), International Society for Heart and Lung Transplantation (ISHLT). Eur Respir J 2015;46:903-75. Erratum in: Eur 
Respir J. 2015 Dec;46(6):1855-6.

2. Delcroix M, Lang I, Pepke-Zaba J, et al. Long-Term Outcome of Patients With Chronic Thromboembolic Pulmonary Hypertension: Results From an International Prospective Registry. Circulation 2016;133:859-71.

3. Cannon JE, Su L, Kiely DG, et al. Dynamic Risk Stratification of Patient Long-Term Outcome After

Cite this article as: Palazzini M, Saia F, Taglieri N, Guarino D, Rotunno M, Galiè N, Dardi F. Balloon pulmonary angioplasty after pulmonary thromboendarterectomy. Ann Cardiothorac Surg 2022;11(2):192-194. doi: 10.21037/acs-2021-pte-19
Pulmonary Endarterectomy: Results From the United Kingdom National Cohort. Circulation 2016;133:1761-71.

4. Shimura N, Kataoka M, Inami T, et al. Additional percutaneous transluminal pulmonary angioplasty for residual or recurrent pulmonary hypertension after pulmonary endarterectomy. Int J Cardiol 2015;183:138-42. 